JOURNAL OF

APPLIED

CRYSTALLOGRAPHY

ISSN 1600-5767

\section{Detailed surface analysis of V-defects in GaN films on patterned silicon(111) substrates by metal- organic chemical vapour deposition. Corrigendum}

Received 6 January 2020

Accepted 7 January 2020

Keywords: gallium nitride; threading

dislocations; transmission electron microscopy
Jiang-Dong Gao, Jian-Li Zhang,* Xin Zhu, Xiao-Ming Wu, Chun-Lan Mo, Shuan Pan, Jun-Lin Liu and Feng-Yi Jiang

National Institute of LED on Silicon Substrate, Nanchang University, Nanchang, Jiangxi 330096, People's Republic of China. *Correspondence e-mail: zhangjianli@ncu.edu.cn

An error in the article by Gao, Zhang, Zhu, Wu, Mo, Pan, Liu \& Jiang [J. Appl. Cryst. (2019), 52, 637-642] is corrected.

A label error has been found in Fig. 3(d) in the paper by Gao et al. (2019). The error is that 'Terrace High GR' should read 'Terrace Low GR'. Here we publish the revised Fig. 3. This correction does not affect the text description in the paper.

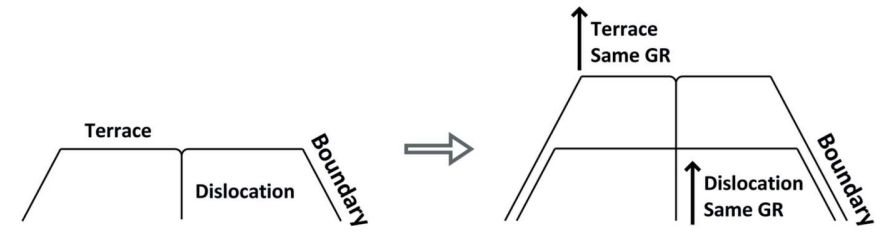

(b)

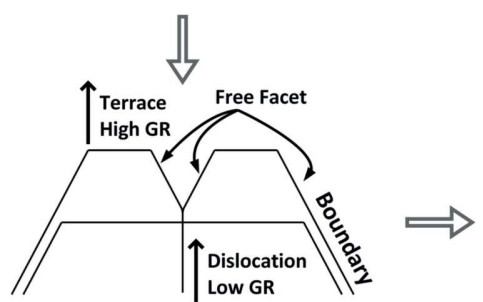

(c)

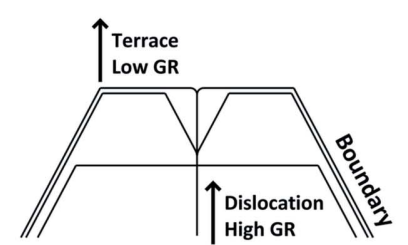

(d)
Figure 3 (revised)

Schematic representation of the growth mode of a V-defect. (a) Original state, $(b)$ normal growth, $(c)$ formation of the V-defect and (d) filling up of the V-defect.

\section{References}

Gao, J.-D., Zhang, J.-L., Zhu, X., Wu, X.-M., Mo, C.-L., Pan, S., Liu, J.-L. \& Jiang, F.-Y. (2019). J. Appl. Cryst. 52, 637-642. 